\title{
Una mirada al futuro de la tecnología $y$ del ser humano. Entrevista con Anders Sandberg
}

\author{
Looking to the Future of Technology \\ and Human Being. \\ An Interview with Anders Sandberg
}

\author{
ANDERS SANDBERG \\ Oxford University
}

ANTONIO DIÉGUEZ

Universidad de Málaga

(Autor de la entrevista, la traducción y la introducción)

Recibido: 28-IX-15 Aceptado 20-X-15

\section{RESUMEN}

Entrevista realizada a Anders Sandberg, miembro del Future of Humanity Institute de la Universidad de Oxford y experto en mejoramiento humano y transhumanismo, sobre cuestiones centrales de su labor investigadora.

\section{PALABRAS CLAVE}

TRANSHUMANISMO, MEJORAMIENTO HUMANO, ANDERS SANDBERG, BIOTECNOLOGÍA

\section{ABSTRACT}

Interview with Anders Sandberg, member of the Future of Humanity Institute at Oxford University and expert in human enhancement and transhumanism, about central topics in his works.

KEYWORDS

TRANSHUMANISM, HUMAN ENHANCEMENT, ANDERS SANDBERG, BIOTECHNOLOGY 


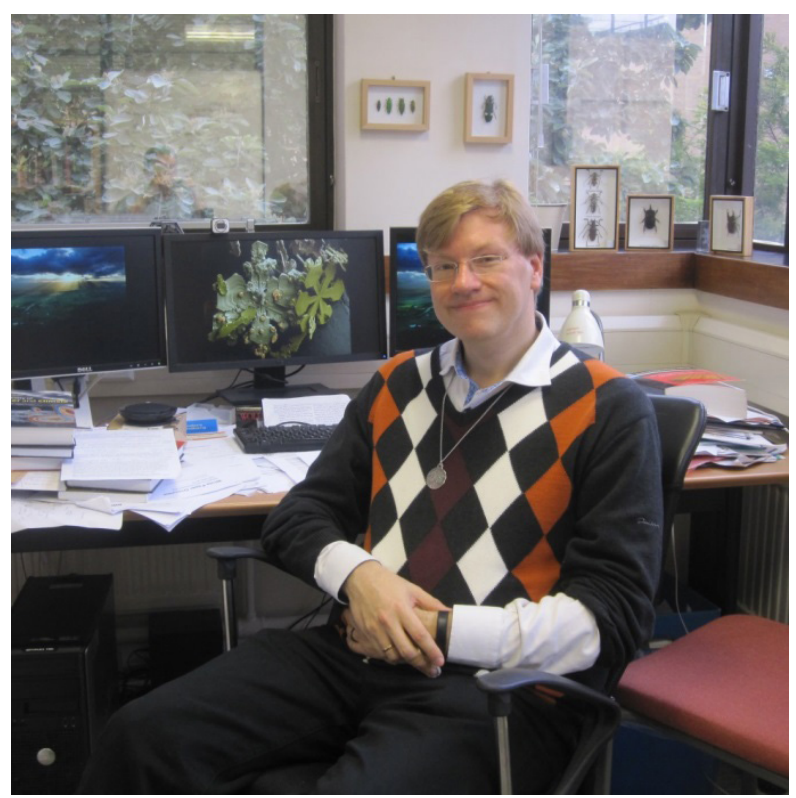

La caracterización más concisa y más ajustada que conozco de Anders Sandberg la escribió en un libro el filósofo de la Universidad de Duke, Allen Buchanan, y dice así: «Sandberg es un genio multifacético, un filósofo, neurocientífico, futurista matemático y artista del diseño por computador que trabaja en el Centro Uehiro para la Ética Práctica de Oxford».1

Pocos filósofos se atreven ciertamente a mirar hacia el futuro con la audacia y la seguridad con la que él lo hace. Se podrá estar más o menos de acuerdo con sus ideas, sus análisis o sus previsiones, pero lo que no se puede negar es que siempre están fundados en sólidos conocimientos científicos y en profundas reflexiones filosóficas. En todo caso, él mismo admite que muchas de sus tesis son meramente exploratorias, y no pretenden ni mucho menos pasar por profecías, ni dar por sentado que el futuro está predeterminado de algún modo misterioso. Más bien su idea de fondo parece ser que el futuro está en nuestras manos y que eso precisamente es lo que nos permite abrigar un cierto optimismo acerca de él. Aunque esa convicción no le impide analizar con detalle los posibles riesgos existenciales que nos amenazan, algunos de ellos muy graves. Su confianza en la tecnología es, sin embargo, lo suficientemente grande como

1 Buchanan, A. (2011), Better than Human, Oxford: Oxford University Press, p. 94. En estos momentos, como se dirá a continuación, Sandberg trabaja en el Institute for the Future of Humaniy en Oxford, aunque mantiene lazos estrechos con el Centro Uehiro. 
para no considerarse vencido de antemano. Él se cuenta decididamente en las filas de los que consideran que el desarrollo tecnológico es el mejor recurso que tenemos para afrontar con éxito ese futuro.

Anders Sandberg es desde 2007 miembro del Future of Humanity Institute, de la Universidad de Oxford. Allí investiga -en estrecha colaboración con Nick Bostrom, con el que ha publicado diversos trabajos conjuntos- sobre cuestiones éticas, sociales y políticas relacionadas con las nuevas tecnologías y muy especialmente sobre las tecnologías de mejoramiento humano (mejoramiento cognitivo, mejoramiento emocional, etc.). También centran su interés las indagaciones sobre el modo de prevenir y evaluar los riesgos globales catastróficos que pueden aguardarnos en el futuro, por baja que sea su probabilidad, y sobre diversos aspectos de la neurociencia cognitiva y de la neuroética. Estudió ciencias de la computación en la Universidad de Estocolmo, y se doctoró en esa misma universidad en neurociencia computacional. Es cofundador y director del think tank sueco Eudoxa, dedicado a reflexionar sobre el transhumanismo desde una perspectiva progresista, y entre 1996 y 2000 fue el presidente de la Asociación Transhumanista Sueca. Sus diseños por ordenador han sido también utilizados como ilustraciones para las portadas de algunos libros del escritor australiano de ciencia-ficción Damien Broderick.

Anders Sandberg mantiene un blog sobre asuntos variados, pero a menudo relacionados con el significado de las nuevas tecnologías, cuya visita merece mucho la pena (http://aleph.se/andart2/). Buena parte de sus trabajos académicos y algunos vídeos interesantes pueden verse en su página de la Oxford Martin School: http://www.oxfordmartin.ox.ac.uk/people/49

La entrevista que aquí se publica tuvo lugar durante mi estancia de investigación en el Oxford Uehiro Centre for Practical Ethics en los meses de abril, mayo y junio de 2015. Anders me recibió en su acogedor despacho, situado en una esquina con grandes ventanales en el piso primero del moderno edificio en St. Ebbe's Street que el Future of Humanity Institute comparte con el Uehiro Centre, una mañana de primeros de junio. Me había causado muy buena impresión en alguna reunión en el Uehiro unos días antes y eso, unido a mis lecturas previas sobre su trabajo, me decidió a visitarle. Después de una breve presentación y de una interesante charla sobre el transhumanismo y el impacto medioambiental de las nuevas tecnologías, cobijados por las varias pantallas de ordenador, todas ellas encendidas, que ocupan casi al completo el espacio de su mesa, le pasé el cuestionario por escrito y en el plazo de unos diez días tenía sus respuestas en mi buzón de correo electrónico. Ni que decir tiene que le estoy muy agradecido por la amabilidad y buena disposición con la que me recibió y por el interés que puso en la tarea de contestar a mis preguntas. Creo que el resultado será de gran interés para todo el que se haya introducido alguna 
vez en los debates recientes acerca del transhumanismo y de las tecnologías de mejoramiento.

1. Usted es uno de los autores que más se ha destacado en la defensa del transhumanismo y de la mejora cognitiva, así como de otros tipos de biomejoramiento, desde planteamientos filosóficos. ¿Cómo definiría usted el transhumanismo y a qué atribuye el éxito mediático que está teniendo este movimiento en la actualidad? ¿Cree que es básicamente una corriente filosófica o considera, por el contrario, que encierra otros aspectos más amplios, como por ejemplo, agendas de tipo religioso o político?

Yo definiría el transhumanismo como la idea de que la condición humana no es inmutable, que puede y debe ser cuestionada y cambiada, y que para hacer esto podemos hacer uso de la razón aplicada.

El transhumanismo es en muchos aspectos el fruto del proyecto humanista de mejora de la condición humana, pero amplificado por la comprensión que hoy tenemos de que el cuerpo y la mente son objetos que pueden ser en gran medida entendidos y cambiados tecnológicamente. Sin embargo, el transhumanismo está también abierto a la posibilidad de que pueda haber modos posthumanos de existencia que posean gran valor y de que, por tanto, sea deseable explorar el ámbito posthumano para encontrarlos. Probablemente, ser humano no es el mejor estado posible de existencia.

¿Por qué les gusta tanto el transhumanismo a los medios de comunicación? En parte, probablemente, por la naturaleza transgresora de la idea: desafiar la condición humana suscita emociones fuertes, y a los medios de comunicación les encanta eso. Hay también en él elementos de tecnología puntera y escenarios de ciencia ficción. Todo esto favorece el entretenimiento, aunque no el debate racional.

Y lo que es más importante, el transhumanismo es uno de los pocos movimientos actuales que articula una visión positiva del futuro. En contraste con muchas ideologías del presente, argumenta que las cosas pueden llegar a ser mucho mejores y que aún hay horizontes abiertos por explorar.

¿Es el transhumanismo un movimiento político? Creo que se puede retrotraer el pensamiento transhumanista a la insatisfacción con la condición 
humana y a la conclusión de que debemos dar pasos para cambiarla realmente. Esto es diferente de las opiniones apologéticas (ya sean religiosas o existencialistas) que sostienen que la condición humana es buena o debe ser aceptada, o de las opiniones pesimistas que afirman que la condición humana no puede cambiarse. Pero el optimismo transhumanista no debe ser fundamentado en profundas razones filosóficas particulares. Yo creo, como filósofo, que es bueno considerar cuidadosamente las razones para las propias creencias, pero es fácil encontrar transhumanistas que simplemente han decidido adoptar esa visión.

A veces se ve al transhumanismo como una idea religiosa, y si bien presenta solapamientos con muchas religiones en su preocupación por escapar de la condición humana, se dan también diferencias prácticas y no hay una teoría unificada de los valores que lo abarque. Recientemente he realizado una revisión de las concepciones transhumanistas sobre el significado de la vida ${ }^{2} \mathrm{y}$ quedé impresionado por la diversidad de opiniones. Pude encontrar ejemplos de casi cualquiera de los enfoques más conocidos sobre la cuestión, desde el existencialismo al perfeccionismo o al cristianismo, y también otros más exóticos. Creo que esto pone de evidencia que el transhumanismo en general no posee una teoría unificada de los valores: las tecnologías en discusión son instrumentos para alcanzar fines mejoradores, pero por qué se considera que estos mejoramientos son deseables es algo que depende de teorías sobre el valor y los fines que son más específicas. Se puede hablar de transhumanismos particulares: transhumanismo existencialista, transhumanismo extropiano, transhumanismo cosmista, transhumanismo budista, etc.

Del mismo modo, el transhumanismo encaja bien con diversas agendas políticas: hay transhumanistas liberales (libertarian), socialdemócratas y anarquistas, y cada uno de ellos combina el cuestionamiento transhumanista optimista de la condición humana con teorías políticas acerca de cómo puede y debe cambiarse la sociedad en la práctica. Pero no hay una política inherente al transhumanismo, excepto en lo que se refiere al rechazo de ciertas posiciones conservadoras.

\section{Forma usted parte del personal académico del Future of Humanity} Institute de la Universidad de Oxford. ¿Podría explicarnos brevemente cuál es la finalidad del Instituto y a qué se ha dedicado su investigación dentro de él?

El objetivo del Future of Humanity Institute es ante todo el mejoramiento del futuro de la humanidad. Su propósito es ofrecer una imagen amplia del

2 Anders Sandberg (2014), «Transhumanism and the meaning of life». In Transhumanism and Religion: Moving into an Unknown Future, eds. Tracy Trothen and Calvin Mercer, Praeger. 
futuro de la humanidad a largo plazo. Esto incluye pensar sobre las amenazas a nuestra supervivencia (riesgos existenciales) y sobre las tecnologías emergentes que podrían cambiar la condición humana. También estudiamos cómo pensar racionalmente acerca del futuro y de estos asuntos con un alto grado de incertidumbre.

Formamos parte de la Oxford Martin School, fundada en el 2005 por James Martin para ayudar a resolver los problemas más graves del siglo XXI. La $O M S$ incluye institutos y proyectos acerca de casi todos los grandes temas, desde el cambio climático a la guerra, desde la nanomedicina al envejecimiento. Nuestro papel es mirar lejos y centrarnos en las cosas que significarían una gran diferencia en los valores que repercuten en el modo en que debemos actuar.

El Instituto no es en sí mismo transhumanista, pero nos ocupamos de muchas cuestiones que están estrechamente ligadas a la indagación transhumanista sobre la condición humana.

Mi propia investigación en el Instituto se ha centrado en la tecnología, la ética y el impacto social del mejoramiento cognitivo y emocional. También he trabajado sobre cuestiones de riesgos e incertidumbres, especialmente cuando se trata de sistemas tecnológicos complejos, cognición colectiva y riesgos globales. Finalmente, he investigado sobre los límites físicos dentro de los cuales la vida y la inteligencia podrían ejercer influencia sobre el universo.

3. Las promesas del transhumanismo son muy arriesgadas: una vida de longitud indefinida, superación de todas las enfermedades y de los límites corporales que nos han caracterizado como especie, adquisición de rasgos completamente nuevos y ajenos a nuestra condición natural, unión con la máquina, volcado de nuestra mente en superordenadores, etc. Estas promesas han despertado un gran entusiasmo en algunos, pero en general han sido recibidas con escepticismo. ¿Cree usted que todas ellas están justificadas? ¿Confía realmente en que la mayor parte podrán ser cumplidas en el futuro? Y en caso de que sea así, ¿cree que eso es deseable, dado que probablemente significaría la extinción de nuestra especie biológica? ¿No le preocupa que estemos preparando el camino para nuestro propio final?

Los transhumanistas no son ni mejores ni peores que cualquier otra persona en lo que a la predicción del futuro se refiere. No obstante, el objetivo es más bien motivar al mundo a que construya un futuro deseado, y no tanto realizar promesas acerca de lo que ha de sucederá en dicho futuro.

Cuando dirigen su mirada a la tecnología, los transhumanistas se entusiasman con lo sucedido en los últimos 30 años, en los cuales los progresos han sido interesantemente desiguales. 
Los medicamentos mejoradores de la cognición son usados por estudiantes y profesionales, aunque sus efectos reales son relativamente limitados. El progreso futuro depende aquí más de la situación que se dé en la regulación legal que de la ciencia misma. Otras formas de mejoramiento cognitivo, tales como la estimulación cerebral, la neuro-retroalimentación (neurofeedback) y el aprendizaje mediante juegos de ordenador, han mostrado ser mucho más prometedoras. Pese a los impresionantes avances en optogenética y nanotecnología, las interfaces cerebro-ordenador están aún a décadas de poder ser lo suficientemente simples y seguras como para que sean usadas por las personas sanas que deseen hacerlo.

La biotecnología ha avanzado a pasos agigantados. Es ciertamente impresionante lo que podemos hacer en la actualidad con las células madre, la secuenciación genética, la biología sintética, la síntesis de ADN, y la edición de genes; y es probable que estas capacidades actuales sean mucho más poderosas en un futuro próximo. Sin embargo, hay aún una brecha significativa entre el laboratorio y la clínica. La terapia génica se estancó, y solo ahora parece retornar. El uso reciente de la técnica de edición genómica CRISP/cas9 para modificar embriones humanos ha sido un paso radical, aunque se ha puesto de manifiesto que aún se necesita mucha más investigación al respecto.

Las tecnologías de la información se han desarrollado quizás de forma más radical de lo que los transhumanistas de los 90 pudieron sospechar: no sólo tiene hoy acceso a Internet el 40\% de la humanidad (una curva que crece rápidamente), sino que las funciones que nos posibilita la moderna Red incluyen nuevas formas de organización social y de medios de comunicación, permiten una diseminación sorprendentemente potente del conocimiento (Wikipedia, MOOCs [Massive Open Online Courses], motores de búsqueda), y proporcionan datos que están conduciendo a una segunda revolución en el aprendizaje de las máquinas, la robótica y la inteligencia artificial. De hecho, la preocupación de muchos transhumanistas es que podamos tener formas demasiado poderosas de automatización y de IA antes de que dispongamos de buenos métodos para controlarlas.

La extensión de la vida es otro caso en el que están produciéndose progresos científicos muy rápidos en biogerontología que, sin embargo, se están transfiriendo a la práctica clínica mucho más lentamente de lo que se esperaba (para disgusto inmenso, claro está, de los transhumanistas de edad avanzada). Una cuestión clave aquí es que la financiación de la investigación médica tiende a centrarse en el tratamiento de los efectos (en las enfermedades producidas por el envejecimiento) antes que en el de las causas.

La lista podría prolongarse con las tecnologías espaciales, la nanotecnología, el dinero digital, y muchas otras tecnologías. 
Mi conclusión es que hay suficientes tecnologías prometedoras para sostener como virtualmente cierta la afirmación de que los seres humanos del futuro habrán sido mejorados: incluso si algunas de estas tecnologías fracasan o experimentan un desarrollo lento, hay en juego las suficientes como para que alguna de ellas tenga éxito. Solo que aún no sabemos cuál.

Muchas de las tecnologías que han tenido un desarrollo decepcionante se han visto también ralentizadas por razones sociales, económicas o culturales y no tanto por imposibilidades científicas subyacentes. El transhumanismo, como movimiento cultural, es por supuesto un planteamiento más apropiado para comprometerse en estas cuestiones que la simple investigación directa, si bien hay bastantes científicos y empresarios motivados por el transhumanismo.

¿Es el transhumanismo el final de nuestra especie? Como es bien sabido, Francis Fukuyama lo calificó de la idea más peligrosa del mundo. El núcleo de su inquietud -y de la de muchos otros-con el transhumanismo es que éste amenaza algún tipo de esencia humana, y sin esa esencia la sociedad o la humanidad no pueden existir.

Obviamente, el problema consiste en definir esa esencia de un modo que no sea tan parroquiano como lo fueron en el pasado las discriminaciones morales contra las mujeres, otras razas, otros tipos de sexualidad o de cerebro, al tiempo que no sea tan general (por ejemplo, 'ser capaz de agencia moral') que los posthumanos puedan poseerla. Incluso si algunos humanos mejorados se llegaran a convertir en una nueva especie en virtud de sus diferencias fundamentales con la nuestra, no está claro que esto haya de tener alguna importancia moral, a menos que pierdan aspectos fundamentales de la agencia moral, algo bastante improbable.

Si el Homo sapiens actual se extinguiera a causa de su transformación gradual en una nueva especie (¿Homo excelsior?), esta pérdida se asemejaría a la pérdida experimentada por Homo erectus en su evolución hacia Homo sapiens. Es posible que fuera una pequeña pérdida de especies y de diversidad cultural, pero fue compensada por las grandes ventajas que portaban los miembros de la nueva especie. Podríamos desear que los humanos del futuro se desplieguen en una multitud de formas y especies de modo que ganemos el valor de la diversidad y la variedad además de los beneficios que les acompañen.

Sin embargo, cabe esperar que las tecnologías poderosas sean peligrosas también si son usadas de forma imprudente. La tecnología del mejoramiento mental podría convertirse en la tecnología del control mental; no hay ninguna garantía de que los sistemas superinteligentes tengan fines compatibles con los de los seres humanos; el control sobre la biología o la materia podría dar rienda suelta a nuevas y devastadoras formas de armamento, etc. Esta es la razón por la que los transhumanistas, quienes después de todo tienden a tomarse en serio el potencial de la tecnología radical del futuro, están también involucrados en 
los esfuerzos por encontrar modos de hacer que tales tecnologías posibles sean más seguras en función de su diseño, antes de que estén en funcionamiento. Podríamos no tener una segunda oportunidad si dejamos este análisis hasta que las nuevas súper-armas sean ya una certeza virtual.

4. ¿Debería haber límites para estas transformaciones? Los críticos del transhumanismo no solo señalan problemas éticos, sino también de tipo político y social, como el incremento de las desigualdades, el peligro de un mayor control sobre los individuos y la creación de dos comunidades enfrentadas, la de los humanos y la de los posthumanos. ¿Cree que tienen razón en esas preocupaciones politico-sociales?

Siempre hay límites éticos, prácticos y sociales al mejoramiento, pero yo no creo que sean tan restrictivos como muchos críticos piensan.

Mi posición de base es que tenemos derecho a la libertad morfológica: se nos debe permitir cambiar -o no cambiar-nuestro cuerpo y nuestra mente. Esto es una consecuencia de la autonomía básica, o, en el marco de los derechos, es una consecuencia de nuestro derecho a la libertad y a nuestro propio cuerpo.

Sin embargo, esta libertad está constreñida por nuestra capacidad-hemos de ser completamente consciente de lo que estamos haciendo-y por el daño que el cambio podría causar a la libertad de otros. Pero que a esos otros puedan no gustarles nuestros cambios no es motivo suficiente para prohibirlos. Tendrían que ser activamente perjudiciales para ellos. Y en un mundo en el que está permitido conducir coches esto es poner el umbral alto.

Se puede argüir que ciertas mejoras nos cambiarían tanto que el resultado sería una nueva persona; otras mejoras podrían incluso convertirnos en nopersonas (y presumiblemente ambas cosas despertarían desaprobación moral). Sin embargo, es poco probable que muchas personas busquen tales cambios. La gente está mucho menos dispuesta a mejorar lo que considera capacidades centrales del yo, tales como la empatía y la personalidad, que otras capacidades menos orientadas al yo, como la atención, la memoria y las habilidades lingüísticas. ${ }^{3}$

Las consideraciones socio-políticas tienen diferente peso dependiendo de la filosofía política de cada uno. Una visión liberal clásica como la que se ha esbozado antes no aceptaría que la desigualdad sea una razón válida para limitar el mejoramiento. Una visión más socialdemócrata pensará, claro está, que un mejoramiento que incremente la desigualdad es problemático (aunque

3 Riis, J., Simmons, J. P., \& Goodwin, G. P. (2008), «Preferences for enhancement pharmaceuticals: The reluctance to enhance fundamental traits», Journal of Consumer Research, 35(3), 495-508. 
se puede adoptar un enfoque rawlsiano y argüir que ese tipo de mejoramientos podría estar justificado si el beneficio para los menos afortunados es lo suficientemente grande).

Muchas de estas preocupaciones comunes son susceptibles de un análisis empírico, mejor que uno filosófico. Por lo habitual, los medicamentos y los artefactos tienen precios que bajan exponencialmente con el tiempo; por lo tanto, los mejoramientos basados en medicamentos o en artefactos probablemente serán baratos y estarán extendidos. Por otro lado, los servicios se mantienen siempre caros cuando no pueden ser automatizados, y los mejoramientos que estén basados en los servicios probablemente presentarán un riesgo mayor de tener malos efectos sociales. Con todo, sabemos que algunos servicios, como las escuelas y la salud, son percibidos como algo tan esencial para la sociedad (la educación básica es incluso obligatoria en muchos países) que son sufragados mediante impuestos a pesar de sus altos costes. Parece plausible pensar que aquellos tipos de mejoramiento que tuvieran un impacto significativo en nuestras vidas terminen formando parte de una categoría similar.

Cuando analizamos el mejoramiento como algo que una sociedad podría realmente hacer, en lugar de como una posibilidad futura sobre la que filosofar, entonces el asunto se vuelve más manejable. Podemos considerar si el pago de impuestos tendría efectos beneficiosos en la adopción del mejoramiento, podemos considerar cómo mantener la privacidad, el derecho al acceso, o los informes sobre seguridad-consideraciones que nos son ajenas a nuestro discurso sociopolítico normal. Existe el peligro de convertir el mejoramiento en una pantalla abstracta sobre la que proyectar nuestros miedos y nuestras esperanzas, dado que esta forma de discusión no permite habitualmente la aplicación del gran número de útiles herramientas éticas, sociales y políticas que poseemos.

5. Acerca del modo de afrontar el cambio climático, usted ha afirmado en un artículo conjunto ${ }^{4}$ que más fácil y menos arriesgado que la geoingeniería es la bioingeniería encaminada a modificar al ser humano para hacerlo menos destructivo de los recursos del planeta o más adaptado a las nuevas situaciones ambientales creadas por dicho cambio climático. A esto cabría objetar, sin embargo, que incluso si la ingeniería aplicada al ser humano fuera más segura que la geoingeniería, habría que intentar antes otras soluciones, como las que proponen los ecologistas, que conduzcan a un modo de vida

4 [El artículo en cuestión es el siguiente: S. Matthew Liao, Anders Sandberg \& Rebecca Roache (2012), «Human Engineering and Climate Change», Ethics, Policy \& Environment, 15:2, 206-221, DOI: 10.1080/21550085.2012.685574. Nota de A. D.]. 
con menos impacto en el planeta. Al fin y al cabo son determinados sistemas económicos y políticos los que constituyen la causa principal del problema.

Merece la pena subrayar que ese artículo intentaba justamente explorar por qué el cambio de los sistemas económicos y políticos habría de ser la prioridad. El argumento común entre los ecologistas parece ser que las soluciones que van a favor de la corriente, como la geoingeniería o el aprender a vivir con un clima transformado, están mal, mientras que las soluciones a contracorriente, como cambiar la economía, están bien. ¿Es debida la bondad de éstas últimas al hecho de que van a contracorriente, acercándose a las causas últimas? Se podría decir que nuestro argumento constituye una reductio ad absurdum de ese planteamiento. En última instancia, mi propia valoración es que [el argumento expuesto en el artículo] muestra cuanto menos que es necesario tener en cuenta consideraciones éticas no-ecológicas a la hora de ofrecer soluciones a los problemas creados por el cambio climático, y que debemos estar más abiertos a soluciones no estándar.

El discurso sobre el clima a menudo asume implícitamente que, puesto que el clima es un problema global de enorme importancia, inmediatamente tiene prioridad sobre otras consideraciones, pero esto sobrevalora su importancia. Los riesgos existenciales, las amenazas para toda la humanidad futura, tienen ciertamente una enorme importancia moral. ${ }^{5}$ Pero el cambio climático probablemente solo reducirá nuestro bienestar futuro. No es una amenaza para nuestro potencial a largo plazo; es importante, pero no de una importancia predominante. ${ }^{6}$

Podría parecer sorprendente que los transhumanistas, que después de todo son tachados a menudo de exorbitantemente optimistas acerca del futuro, estén también profundamente interesados en los riesgos existenciales. Pero esta preocupación se sigue naturalmente de su cuestionamiento de la condición humana y de su optimismo sobre el futuro. Si la condición humana puede cambiar de forma profunda, podría cambiar a peor, e incluso desaparecer. La tesis transhumanista de que la condición humana puede verse influida por nuestras acciones implica que somos responsables de ella. Si el futuro puede traer valores enormemente importantes -y eso lo creen la mayor parte de los transhumanistas-, entonces las amenazas para la consecución de estos valores

5 Bostrom, N. (2013), «Existential risk prevention as global priority». Global Policy, $4(1), 15-31$.

6 Hay una probabilidad relativamente baja, aunque no igual a cero, de que sea una amenaza existencial debido a riesgos catastróficos extremos normalmente descartados en las discusiones habituales sobre el clima. Esto podría cambiar las cosas, pero el clima sería aún un riesgo existencial entre otros, como la guerra nuclear, las armas biológicas y los impactos de asteroides. 
son también más serias. Por lo tanto, salvaguardar el futuro de esas amenazas accidentales o deliberadas se convierte en una máxima prioridad.

6. En ocasiones parece como si los transhumanistas se hubieran dicho: mejorar el mundo es dificil, busquemos solo mejorarnos tecnológicamente a nosotros mismos, quizás así ya no necesitemos demasiado de este mundo ni, por tanto, tampoco mejorarlo. ¿No le parece que modificar al ser humano, por ejemplo, reduciéndolo de tamaño, para que su consumo sea menor, o adaptarlo a un planeta deteriorado, es asumir que debemos seguir como hasta ahora, sin cambiar nuestra forma de vida, solo porque la tecnología podrá hacernos más tolerantes al calor, a la sequía y al aire y agua contaminados? Esto suena a pensamiento conservador, ¿no cree?

Una crítica común a la propuesta del mejoramiento humano es que éste no resuelve los problemas subyacentes (obsérvese cómo esto es exactamente lo opuesto a la crítica que se ha hecho contra la idea defendida en el artículo mencionado antes sobre la ingeniería aplicada al ser humano). En lugar de hacer que los conductores de camiones, los pilotos o los cirujanos tomen drogas para que estén más alerta, sería preferible que tuvieran horarios de trabajo que les permitieran un descanso adecuado. Pero esto se basa en el supuesto de que el problema subyacente es resoluble: a pesar de que hay riesgos obvios y bien documentados, no se lleva a cabo el descanso en estos oficios por diversas razones prácticas, económicas y culturales. La situación es algo similar a la que se da en las discusiones entre los defensores de la terapia cognitivo-conductual y del psicoanálisis. Mientras que el psicoanálisis podría llevar a las causas que están en la raíz de los problemas y proporcionar una comprensión profunda de los mismos, la terapia cognitivo-conductual puede resolver los problemas prácticos mucho más rápidamente y de forma más barata.

La cuestión es si debemos ocuparnos de las causas profundas o si vamos a resolver los problemas reales. En lugar de erradicar los patógenos infecciosos (la causa), podemos vacunar o usar antibióticos contra ellos de modo que nadie caiga enfermo (que es lo que nos preocupa). Una intuición extendida es que resolver las causas profundas proporciona mejores soluciones, puesto que dichas soluciones son más flexibles y eficaces (un buen ejemplo es el modo en que tanto la terapia cognitivo-conductual como los antidepresivos pueden curar la depresión, pero las estrategias de afrontamiento que se aprenden en la terapia reducen también los riesgos de recaída). Lentificar el envejecimiento reducirá el lastre de muchas enfermedades mucho más de lo que lo harían las curas individuales. Pero muchos otros enfoques son también eficaces pese a no ocuparse de las causas. Las normas sociales y de orden público funcionan 
a la hora de promover una conducta amable sin necesidad de hacer a la gente moralmente perfecta. Por lo tanto, desde una perspectiva consecuencialista, no deberíamos preocuparnos de si una intervención es biotecnológica, psicológica o social, sino que debemos preguntarnos si sus resultados son buenos comparados con los costes y los riesgos implicados.

¿Es difícil mejorar el mundo? No hay una respuesta general a esa pregunta. Muchos transhumanistas, que son optimistas acerca de la tecnología, piensan realmente que muchos de los ominosos problemas de la actualidad -el cambio climático, la escasez de recursos, la mala gobernanza- pueden ser resueltos por medio de la tecnología y/o mediante cambios sociales. Pero tener un plan $\mathrm{B}$-cuerpos más resistentes, adaptación al clima, una vida más eficiente con los recursos, organizaciones sociales ocultas-mejora claramente la situación. Que exista un plan alternativo no quiere decir que sea el más deseable. La mayor parte de los transhumanistas prefieren un planeta sano, rico y libre a cualquier alternativa en la que un plan B deba compensar las cosas malas que nos sucedan.

Creo que hay una arraigada tendencia a suponer que el riesgo moral es más significativo de lo que realmente es. Esto lleva a los conservadores a prohibir las vacunas contra el virus del papiloma humano o los anticonceptivos porque piensan que podrían dar lugar a una mayor promiscuidad; lleva igualmente a los ecologistas a argumentar en contra de la investigación en geoingeniería o acerca de la adaptación a los cambios climáticos, y lleva a algunos comités de ética a rechazar los estudios sobre los efectos reales de los potenciadores cognitivos en estudiantes que los están tomando ya de hecho, puesto que cualquier resultado positivo podría ser interpretado como un respaldo a su uso. Estas preocupaciones se centran en el problema equivocado e intentan normalmente impedir que sepamos más acerca de nuestras opciones posibles.

7. ¿Cree usted que las personas que son jóvenes ahora podrán acceder a estas tecnologías y que, por tanto, algunas de ellas serán los futuros posthumanos?

Incluso los modelos demográficos conservadores sugieren que un gran número de niños pueden tener hoy ya una esperanza de vida de 100 años. Dado que ya estamos utilizando el mejoramiento en alguna medida -desde las drogas inteligentes a los teléfonos inteligentes-y que el mejoramiento será radicalmente mejor con el tiempo (incluyendo la extensión de nuestra esperanza de vida), cabe esperar que estos niños tengan una buena oportunidad de convertirse en posthumanos. Como señaló el autor de ciencia-ficción Charles Stross, la generación que está creciendo justo en este momento, «nunca estará sola, nunca estará perdida, nunca olvidará». 
La pregunta interesante es, claro está, cuánto de radicalmente mejorado o de posthumano puede alguien llegar a ser, sin que eso lo haga una persona diferente. ¿Tiene sentido hablar de «mi» yo posthumano, si tal ser es tan enormemente diferente de mí que mi contribución actual a su identidad es menor que la contribución que hace la tecnología? Mi propia opinión es que la continuidad importa en alguna medida: un posthumano altamente mejorado podría recordar aún su «infancia» humana, del mismo modo que nosotros podemos recordar vagamente nuestra infancia temprana -cuando éramos aún seres que tenían muy poco en común con el yo que somos en el presente.

Sin embargo, la identidad personal podría estar sobrevalorada como guía. Podría ser más importante que el futuro esté poblado de seres con una vida excelente y llena de significado que el hecho de que alguno de ellos sea la continuación de nosotros mismos.

8. Hay un cuento de Asimov, titulado "La última pregunta», en el que una computadora que se autoperfecciona y a la que se le pregunta reiteradamente a lo largo de la historia de la humanidad si es posible revertir la entropía en el Universo, siempre contesta que no tiene datos suficientes para una respuesta definida, hasta que finalmente, trillones y trillones de años en el futuro, incluso después de la muerte de todo el Universo, transformada ya en una entidad que no es materia ni energía, encuentra la respuesta y añade a continuación «hágase la luz». ¿Cree que el posthumano puede aspirar a una situación de trascendencia espiritual similar a la que el cristianismo y otras religiones prometen como la existencia después de la muerte? ¿Puede alcanzar el posthumano a través del constante perfeccionamiento el estatus de un dios o de un ángel?

En las discusiones sobre el transhumanismo, cito a menudo el «Discurso sobre la dignidad del hombre» de Pico della Mirandola, el pasaje en el que Dios le dice a Adán que el verdadero don del ser humano es la capacidad para reconfigurar su propia naturaleza:

No te he hecho ni celeste ni terreno, ni mortal ni inmortal, para que como libre y extraordinario artífice y escultor de ti mismo te forjaras en la forma que prefirieras. Podrás degenerar en seres inferiores, que son los brutos; podrás regenerarte, según tu decisión, en entidades superiores, que son divinas.?

7 Traducción de A.D. basada en el texto latino. 
Yo no me tomo en serio la imaginería religiosa de la línea trazada por la Gran Cadena del Ser, sin embargo, creo que la visión de Pico della Mirandola es la correcta. Nosotros nos hacemos a nosotros mismos y podemos llegar a ser mejores en muchos aspectos fundamentales.

Los seres humanos tenemos acceso a ámbitos de actividad y de pensamiento que estarán siempre más allá de la comprensión de otros simios: podemos experimentar el arte, la ciencia, la religión y la filosofía. Podemos reflexionar sobre nosotros mismos y sobre nuestro lugar en el universo, asumiendo la responsabilidad moral de lo que hacemos. Creo que no sería muy inexacto decir que esto es una forma de progreso espiritual, comparándonos con los simios. Pero no hay ninguna razón para pensar que nuestras capacidades son el límite superior que los seres pueden experimentar. Ésa es la razón por la cual explorar el ámbito posthumano es importante. Existen muchos estados o actividades valiosas que ni siquiera podemos comprender como humanos y que son mucho más importantes que cualquier cosa que hoy sepamos.

Hay una diferencia entre el poder sobre el mundo material y el mental, por un lado, y el tener una acertada comprensión y búsqueda de los valores, por otro. Lo primero es meramente instrumental, mientras que lo segundo pertenece al ámbito de lo ético. Muchos transhumanistas creen que necesitamos lo primero para hacernos mejores en lo segundo. Las profundas preguntas filosóficas se nos han resistido durante milenios, así que puede lo que necesitemos sea o bien asegurar nuestra supervivencia hasta que dichas preguntas puedan ser resueltas (quizás dentro de algunos milenios), o bien crear mentes más grandes que puedan ayudarnos a resolverlas. Cuando sepamos mejor a dónde debemos ir, debemos ir allí, pero entretanto mejorarnos en nuestra capacidad de conocer es una buena estrategia.

9. Se ha dicho en ocasiones que los objetivos transhumanistas ocultan una falta de atención a los problemas más graves y urgentes que aquejan hoy día a la humanidad; problemas como el hambre y la pobreza, o las injusticias sociales, que son los que impiden un mejoramiento real y accesible en las condiciones de vida de la mayoría de las personas, y que podrían quizás encontrar algún alivio a través de un mejor uso de la tecnología. No es extraño que precisamente por ello el transhumanismo haya sido acusado de escapismo ante los problemas de los seres humanos actuales, que son los que deberían centrar nuestras preocupaciones. ¿Qué piensa de esta acusación? ¿Cree que es injusta? ¿Cree que el transhumanismo si tiene sensibilidad para estos problemas? Me consta que el Future of Humanity Institute está implicado en actividades humanitarias de diverso tipo. 
Como subrayé antes, existen muchas combinaciones de la mentalidad básica transhumanista y diferentes sensibilidades sociales. Mientras que los transhumanistas liberales (libertarian) pueden pensar que dar a la gente máxima libertad y prosperidad es lo que más contribuirá a mejorar el mundo, los transhumanistas progresistas piensan que la solución vendrá mediante diversas formas de redistribución o ayuda proactiva. Y, por supuesto, hay transhumanistas egoístas que no se preocupan nada de los demás.

Se da la circunstancia de que el Future of Humanity Institute adopta a menudo una perspectiva consecuencialista en ética. Esto ha conducido a que haya un gran solapamiento con el movimiento que se conoce como «altruismo eficaz», que intenta encontrar, basándose en la evidencia empírica, qué intervenciones [humanitarias] tienen el mayor impacto positivo. Las consideraciones filosóficas acerca del valor de las generaciones futuras, el beneficio del análisis cuidadoso y la importancia de pensar a lo grande, encajan bien con el transhumanismo.

Una cuestión importante en la perspectiva social del transhumanismo es cuánto debemos preocuparnos de los problemas actuales si los comparamos con las ganancias que podemos obtener en el futuro. Malgastar en el presente todos nuestros recursos disponibles [en solucionar esos problemas] puede significar que muchos riesgos importantes queden sin atender y que perdamos oportunidades importantes.

Un buen ejemplo lo constituyen los teléfonos móviles. Originalmente no fueron más que juguetes para ejecutivos ricos, y sin embargo su amplia adaptación en Occidente condujo a una caída radical en los precios y a una mejora en sus prestaciones. Hoy, los teléfonos inteligentes se extienden rápidamente en los países en desarrollo, en los cuales tienen un gran impacto en lo que a la pobreza y a la libertad se refiere (sorteando a los monopolios de la información y la carencia de infraestructuras). Si hubiésemos gastado en solucionar la pobreza los recursos que gastamos en la producción de teléfonos móviles a principios de los noventa, el efecto habría sido mucho menor, y al resto del mundo le habría ido también peor.

No todas las innovaciones tienen ese efecto de «todos ganan». Pero muchas tecnologías de interés para los transhumsnistas tendrán probablemente efectos positivos sobre los problemas claves en el mundo. Una mejor salud (especialmente lentificando el envejecimiento) tiene enormes efectos positivos sobre la economía y sobre la capacidad para tener una vida plena. El mejoramiento cognitivo ayudará a estimular el capital humano y la capacidad para resolver los problemas mundiales. La reducción de las restricciones en los recursos gracias al uso de la automatización, la biotecnología o la nanotecnología puede mejorar la resiliencia de las sociedades y el impacto medioambiental. El mejoramiento moral puede incrementar la disposición para arreglar los 
problemas fundamentales. Muchos de estos efectos funcionan en red: hacer a unos pocos más inteligentes, saludables o ricos tiene un impacto menor que hacer a muchos más inteligentes, saludables o ricos, puesto que aquí se aplica la economía de escala.

10. El transhumanismo no oculta que sus fines implican el uso de la eugenesia. Los defensores del transhumanismo aducen que se trata de una «eugenesia liberal», en la que la decisión acerca de qué rasgos han de ser seleccionados estará exclusivamente en manos de los padres $y$ de los individuos, no en manos del Estado o de élites en el poder. ¿Cuál es su opinión sobre este asunto? ¿Está exenta la eugenesia liberal de cualquiera de los problemas morales que se han imputado a la vieja eugenesia?

Yo sí creo que la eugenesia liberal evita los problemas de la vieja eugenesia: no está basada en la coerción estatal, no prescribe un único objetivo de perfección genética, y las razones morales para el mejoramiento son pluralistas en lugar de promover algún fin colectivo.

También creo que el cambio genético de los seres humanos será una cuestión práctica y ética menor que muchas otras (sean o no transhumanistas). La razón es que el tiempo de las generaciones humanas es largo comparado con la velocidad del cambio tecnológico. Durante el tiempo que tarde en crecer y en disfrutar de su condición un niño genéticamente seleccionado o mejorado, es muy probable que otras tecnologías -terapia génica, drogas inteligentes, implantes, nanotecnología, etc.- permitan a una persona no-mejorada de la misma edad disfrutar aproximadamente de los mismos beneficios.

Sin embargo, estas consideraciones ¡no sacan al transhumanismo del apuro! Tal como lo veo, la cuestión ética central en el debate sobre la eugenesia no es la genética, sino que concierne a las pre-personas o personas jóvenes que serán objeto de los cambios. Sospecho que muchos mejoramientos importantes de la salud, la longevidad, la inteligencia y de nuevas capacidades, como las interfaces humano-máquina, habrán de ser instalados en una edad temprana para que puedan ser incorporados adecuadamente por un cuerpo y una mente en desarrollo. Esto tiene la misma importancia ética que el cambio genético directo.

El caso de los cambios muy tempranos, cuando estamos ante una prepersona, creo que es relativamente no problemático (aunque otros discrepan en esto). Coincido con el principio de beneficencia procreativa formulado por Julian Savulescu (si tienes posibilidad de elegir, ceteris paribus, ten el hijo que disponga de las mejores oportunidades para tener una buena vida). El problema comienza cuando consideramos el caso de un niño, que es una 
persona emergente. Aquí las intervenciones afectan a la persona, y es mucho más complejo encontrar el equilibrio entre la autonomía y la beneficencia. No conozco ninguna respuesta ética sucinta acerca de cómo debe manejarse una situación así. Estamos tratando en este caso con el concepto paternidad, un concepto de amplio calado.

Anders SAndBerg es James Martin Research Fellow en el Future of Humanity Institute de la Universidad de Oxford.

Lineas de investigación:

Mejoramiento cognitivo, transhumanismo, riesgos globales, neurociencia cognitiva, neuroética.

Publicaciones recientes:

Ord, T., Hillerbrand, R., SAndberg, A. (2010), «Probing the Improbable: Methodological Challenges for Risks with Low Probabilities and High Stakes», Journal of Risk Research, 13 (2), 191-205.

Liao, S.M., SAndberG, A. (2008), «The Normativity of Memory Modification», Neuroethics, 1 (2) 85-99.

Sandberg, A., Savulescu, J. (2008), «Neuroenhancement of Love and Marriage: The Chemicals Between Us», Neuroethics, 1 (1) 31-44.

Antonio Diéguez es Catedrático de Lógica y Filosofía de la Ciencia en la Universidad de Málaga.

Lineas de investigación:

Realismo científico, filosofía de la tecnología y filosofía de la biología.

Publicaciones recientes:

(2015) «Scientific Understanding and the Explanatory Use of False Models». en M. Bertolasso (ed.) The Future of Scientific Practice: 'Bio-Techno-Logos', London: Pickering \& Chatto Publishers, pp. 161-178.

(2014) «La acción tecnológica desde la perspectiva orteguiana: el caso del transhumanismo», Revista de Estudios Orteguianos, 29, pp. 131-153.

(2013) «When do models provide genuine understanding, and why does it matter?», History and Philosophy of the Life Sciences, 35 (4), pp. 599-620. 